\title{
The Isolation, Identification and Determination of Dehydrotumulosic Acid in Poria cocos
}

\author{
Zonghua Song, ${ }^{*}$ Kaishun BI, ${ }^{* \dagger}$ Xu LuO,* and Kelvin CHAN** \\ *Shenyang Pharmaceutical University, Wenhua Road 103, Shenyang 110016, P. R. China \\ **Research and Development Division, School of Chinese Medicine, Hong Kong Baptist University, \\ Kowloon Tong, Hong Kong
}

\begin{abstract}
Poria cocos (Fuling), a popular Chinese medicinal (CM) herb of fungal origin, has been included in many combinations with other CM herbs for its traditionally claimed activities of inducing diuresis, excreting dampness, invigorating the spleen and tranquilizing the mind and its modern pharmacological use of modulating the immune system of the body. Dehydrotumulosic acid, one of the effective constituents of Fuling, was isolated from the chloroform-soluble material of ethanol extract of the fungus. After further purification by a high-performance liquid chromatographic method on a $\mathrm{C}_{18}$ column, the purified constituent was identified using modern analytical techniques, such as UV, ${ }^{13} \mathrm{C}-\mathrm{NMR}$ and EI-MS. A reversed-phase high-performance liquid chromatographic method has been developed for the determination of dehydrotumulosic acid in Poria cocos. The determination can be accomplished in less than 50 min using methanol-acetonitrile- $2 \%$ glacial acetic acid as the mobile phase at a flow rate of $1.0 \mathrm{~mL} / \mathrm{min}$, with a UV detector setting at $242 \mathrm{~nm}$ and testosterone propionate used as an internal standard. This assay for dehydrotumulosic acid is simple, rapid and with good reproducibility.
\end{abstract}

(Received January 17, 2002; Accepted February 20, 2002)

\section{Introduction}

Traditionally, Poria (Fuling), has frequently been prescribed as one of the chief ingredients in composite prescriptions (Fufang) in traditional Chinese medicine. Nearly $10 \%$ of the traditional Chinese medicinal preparations or preparations admitted to the Chinese Pharmacopoeia (2000 edition) contain Fuling. It is prepared from the fungus of Poria cocos (Schw.) Wolf, known as Fuling in China. It has the traditionally claimed activities of inducing diuresis, excreting dampness, invigorating the spleen and tranquilizing the mind. It was reported that poriatin, the chloroform extract of Poria cocos, has a significant inhibitory effect on the biosynthesis of DNA of leukemia 1210 cells. $^{1}$ However, analytical method available for the quality control of Poria cocos have seldom been reported; perhaps, Tai's report, on a high-performance liquid chromatographic (HPLC) method for dehydropachmic acid determination ${ }^{2}$ is the only one of its kind. The assay presented in this paper is based on a reversedphase HPLC procedure using testosterone propionate as an internal standard. Before finalizing the analytical method we had isolated and identified dehydrotumulosic acid from the extract of Fuling using various analytical tools such as UV, ${ }^{13} \mathrm{C}$ NMR and EI-MS. Subsequently, an assay for the determination of dehydrotumulosic acid in Poria cocos was developed using testosterone propionate as an internal standard by achieving the optimal conditions for chromatographic separation. The method was shown to be adequate for the identification and quantitation of dehydrotumulosic acid in Poria cocos.

\footnotetext{
$\dagger$ To whom correspondence should be addressed.

E-mail: ksbi@mail.sy.ln.cn
}

\section{Experimental}

\section{Materials and reagents}

Poria cocos (Schw.) Wolf samples were collected from different habitats in China. Standard dehydrotumulosic acid was prepared (see following detailed account) and its purity was confirmed as being higher than $98 \%$. The internal standard, testosterone propionate, was ordered from National Institute for the Control of Pharmaceutical and Biological Products, Beijing, China; other chemicals used included: glacial acetic acid, ethanol (95\%), chloroform, petroleum ether and ethyl acetate, all of analytical grade; methanol and acetonitrile, of chromatographic grade.

Extraction, isolation and identification of dehydrotumulosic acid

A quantity of $2 \mathrm{~kg}$ of air-dried powder of Poria cocos was extracted with ethanol. The extract was evaporated to dryness and the residue dissolved with water was extracted with chloroform. The chloroform-soluble fraction $(7.0 \mathrm{~g})$ was chromatographed on a silica-gel column using petroleum ether-ethyl acetate $(\mathrm{v} / \mathrm{v}, 2: 1)$ as the mobile phase to obtain poriatin $(2.0 \mathrm{~g})$. Then poriatin was applied to a semipreparative ODS column $(30 \mathrm{~cm} \times 3 \mathrm{~cm}$ i.d. $)$ and eluted with $\mathrm{CH}_{3} \mathrm{OH}-\mathrm{H}_{2} \mathrm{O}(\mathrm{v} / \mathrm{v}, 7: 1)$ at a flow rate of $10 \mathrm{~mL} / \mathrm{min}$. The main peak, of the retention time is about $18 \mathrm{~min}$, was collected with reference to an UV detector setting at $242 \mathrm{~nm}$. The collected fraction was evaporated and identified by modern analytical techniques, including UV, ${ }^{13} \mathrm{C}-\mathrm{NMR}$ and EI-MS. The UV spectrum showed an absorption maximum at $242 \mathrm{~nm}$. The EIMS spectrum gave the molecular ion $[\mathrm{M}]^{+} 484$, and the fragment ions $[\mathrm{M}-\mathrm{OH}]^{+}$467, $[\mathrm{M}-\mathrm{COOH}-\mathrm{OH}]^{+} 422$ and 


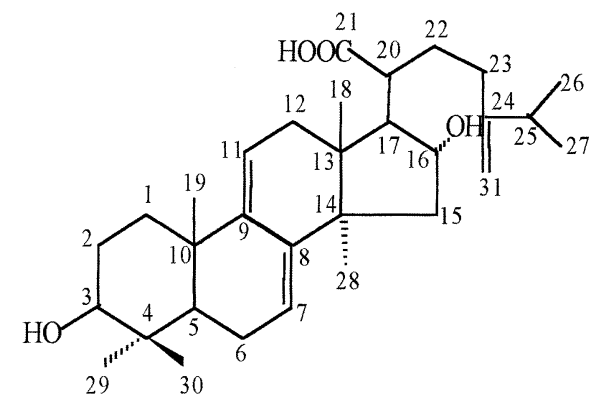

Fig. 1 Molecular structure of dehydrotumulosic acid.

Table 1 Chemical shifts in the ${ }^{13} \mathrm{C}$-NMR spectrum of dehydrotumulosic acid

\begin{tabular}{cccrcr}
\hline $\begin{array}{c}\text { No. of } \\
\text { carbon atom }\end{array}$ & $\begin{array}{c}\delta \text { value, } \\
\text { ppm }\end{array}$ & $\begin{array}{c}\text { No. of } \\
\text { carbon atom }\end{array}$ & $\begin{array}{c}\delta \text { value, } \\
\text { ppm }\end{array}$ & $\begin{array}{c}\text { No. of } \\
\text { carbon atom }\end{array}$ & $\begin{array}{c}\delta \text { value, } \\
\text { ppm }\end{array}$ \\
\hline 1 & 36.7 & 11 & 116.7 & 21 & 179.9 \\
2 & 24.0 & 12 & 30.4 & 22 & 31.1 \\
3 & 82.4 & 13 & 49.3 & 23 & 33.4 \\
4 & 38.4 & 14 & 46.9 & 24 & 156.7 \\
5 & 49.8 & 15 & 44.4 & 25 & 35.0 \\
6 & 19.2 & 16 & 76.7 & 26 & 22.4 \\
7 & 122.0 & 17 & 57.6 & 27 & 23.2 \\
8 & 143.4 & 18 & 17.6 & 28 & 27.5 \\
9 & 147.4 & 19 & 21.5 & 29 & 17.0 \\
10 & 38.5 & 20 & 48.4 & 30 & 26.6 \\
& & & & 31 & 107.4 \\
\hline
\end{tabular}

$[\mathrm{M}-\mathrm{COOH}-2 \mathrm{OH}]^{+}$405. Thus, the molecular structure of the constituent corresponding to the main peak on the chromatogram was identified with that of dehydrotumulosic acid, shown in Fig. 1; this was confirmed by the ${ }^{13} \mathrm{C}-\mathrm{NMR}$ spectrum (Table 1).3,4

\section{Preparation of Poria cocos sample}

An amount of $3.0 \mathrm{~g}$ of dried sample powder of Poria cocos was extracted with $100 \mathrm{~mL}$ of ethanol at $95^{\circ} \mathrm{C}$ in a Soxhlet's extractor for $10 \mathrm{~h}$. The extract was evaporated to dryness, $1 \mathrm{~mL}$ of the internal standard solution $(0.56 \mathrm{mg} / \mathrm{mL})$ added to each sample, and methanol added to make the volume equal to 10 $\mathrm{mL}$.

\section{The chromatographic system}

The HPLC system consisted of a Shimadzu (Tokyo, Japan) LC-10AD pump, an SPD-10A ultraviolet-visible detector, a 20 $\mu \mathrm{L}$ injection loop, and an LC workstation for data collection, all operated at room temperature. The separation column was one filled with hypersile ODS $\mathrm{C}_{18}(200 \times 4.6 \mathrm{~mm}$ i.d., $5 \mu \mathrm{m}$ particle size) and equipped with a $20 \times 4 \mathrm{~mm}$ ODS precolumn. The eluent consisted of methanol-acetonitrile- $0.2 \%$ glacial acetic acid $(13: 12: 10, \mathrm{v} / \mathrm{v})$. The flow rate was $1.0 \mathrm{~mL} / \mathrm{min}$, and the wavelength set at $242 \mathrm{~nm}$.

\section{Calibration by internal standard}

A series of standard solution of dehydrotumulosic acid (0.02, $0.04,0.08,0.16,0.24,0.32$ and $0.40 \mathrm{mg} / \mathrm{mL}$ ) were prepared by diluting the stock solution $(2.0 \mathrm{mg} / \mathrm{mL})$ with methanol. All of the solutions were filtered through a $0.45 \mu \mathrm{m}$ microporous filter, and a $20 \mu \mathrm{L}$ aliquot of the filtered solution was injected into a chromatograph in triplicate. A calibration curve was

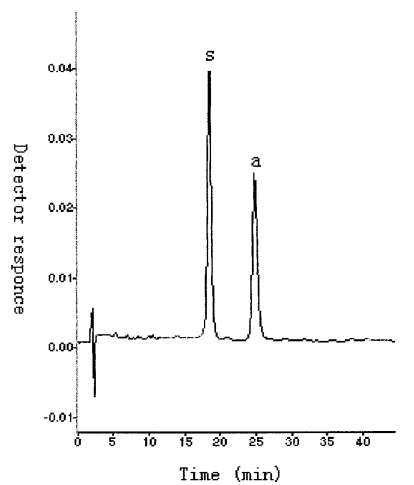

(a)

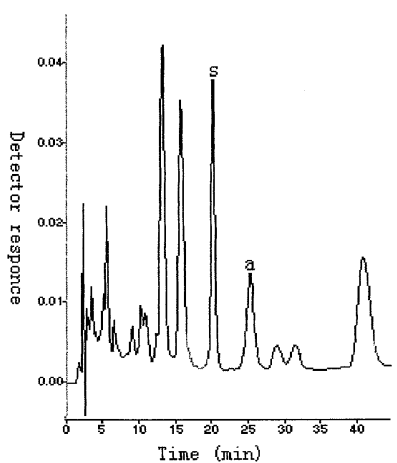

(c)

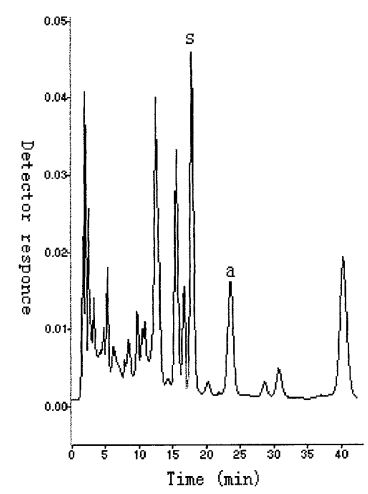

(b)

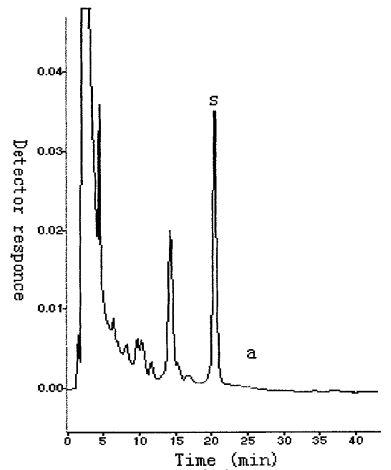

(d)
Fig. 2 Typical chromatograms of dehydrotumulosic acid. (a) Standard dehydrotumulosic acid and internal standard testosterone propionate; (b) a sample from Guilin; (c) a sample from Chengdu; (d) a sample from Xiping. $\mathrm{s}$, testosterone propionate; $\mathrm{a}$, dehydrotumulosic acid.

Table 2 Content of dehydrotumulosic acid in Poria cocos from different habitats of China

\begin{tabular}{clc}
\hline $\begin{array}{c}\text { Sample } \\
\text { No. }\end{array}$ & \multicolumn{1}{c}{ Origin } & $\begin{array}{c}\text { Content of } \\
\text { dehydrotumulosic acid, \% }\end{array}$ \\
\hline 1 & Taihu, Anhui & 0.0786 \\
2 & Enshi, Hubei & 0.2349 \\
3 & Shijiazhuang, Hebei & 0.1754 \\
4 & Guilin, Guangxi & 0.3038 \\
5 & Anqing, Anhui & 0.1860 \\
6 & Lijiang, Yunnan & 0.1936 \\
7 & Qianshan, Anhui & 0.1637 \\
8 & Yuexi, Anhui & 0.1984 \\
9 & Bozhou, Anhui & 0.1682 \\
10 & Emei, Sichuan & 0.1895 \\
11 & Yuexi, Anhui & 0.1964 \\
12 & Chengdu, Sichuan & 0.2644 \\
13 & Hangzhou, Zhejiang & 0.1194 \\
14 & Kunming, Yunnan & 0.1852 \\
15 & Changsha, Hunan & 0.2392 \\
16 & Bozhou, Anhui & 0.1661 \\
17 & Yingshan, Hubei & 0.2461 \\
18 & Xinxiang, Henan & 0.1556 \\
19 & Wuhan, Hubei & 0.0964 \\
20 & Changsha, Hunan & 0.2122 \\
21 & Xiping, Henan & 0.0023 \\
22 & Ling'an, Zhejiang & 0.1489 \\
23 & Gejiu, Yunnan & 0.2155 \\
24 & Shenyang, Liaoning & 0.1066 \\
\hline & &
\end{tabular}


constructed by plotting the peak-area ratio of dehydrotumulosic acid to that of the internal standard against the concentration of dehydrotumulosic acid.

\section{Results and Discussion}

Optimization of extraction time

The effect of the extraction time was examined by allowing the extraction to last $4-12 \mathrm{~h}$. The results showed that an extraction time of $10 \mathrm{~h}$ was necessary for complete extraction.

\section{Evaluation of the assay}

Typical chromatograms of standard dehydrotumulosic acid and internal standard testosterone propionate and three samples of Poria cocos are given in Fig. 2. The calibration curve of the peak-area ratio $(Y)$ versus the concentration $(X, \mathrm{mg} / \mathrm{mL})$ was linear $\left(Y=6.9433 X+0.0346\right.$, the coefficient of determination $r^{2}$ $=0.9999 ; n=7)$. The linear range for the determination of dehydrotumulosic acid was $0.02-0.40 \mathrm{mg} / \mathrm{mL}$. The detection limits was $0.01 \mathrm{mg} / \mathrm{mL}$. The average recovery of dehydrotumulosic acid was $99.9 \%(n=6)$. The repeatability of the assay was $1.4 \%(n=6)$

Determination of dehydrotumulosic acid in Poria cocos

Twenty-four samples of Poria cocos from different habitats in China were analyzed by the proposed method; and the results are shown in Table 2. The proposed HPLC method is simple, accurate, and precise. It needs no pretreatment and provides a new method for the quality control of Poria cocos.

\section{References}

1. J. Xu, D. Lv, and Q. Zhong, Acta Acad. Med. Sin., 1988, 10,45 .

2. T. Tai and A. Akahori, Shoyakugaku Zasshi, 1993, 47, 210.

3. L. A. Cort, R. M. Gascoigne, J. S. E. Holker, B. J. Ralph, A. Robertson, and J. J. H. Simes, J. Chem. Soc., 1954 3713.

4. T. Tai, T. Shingu, T. Kikuchi, Y. Tezuka, and A. Akahori, Phytochemistry, 1995, 39, 1165. 